\title{
Paediatric crossword puzzle 27
}

\section{Manouri P Senanayake ${ }^{1}$}

Sri Lanka Journal of Child Health 2015; 44(2): 121

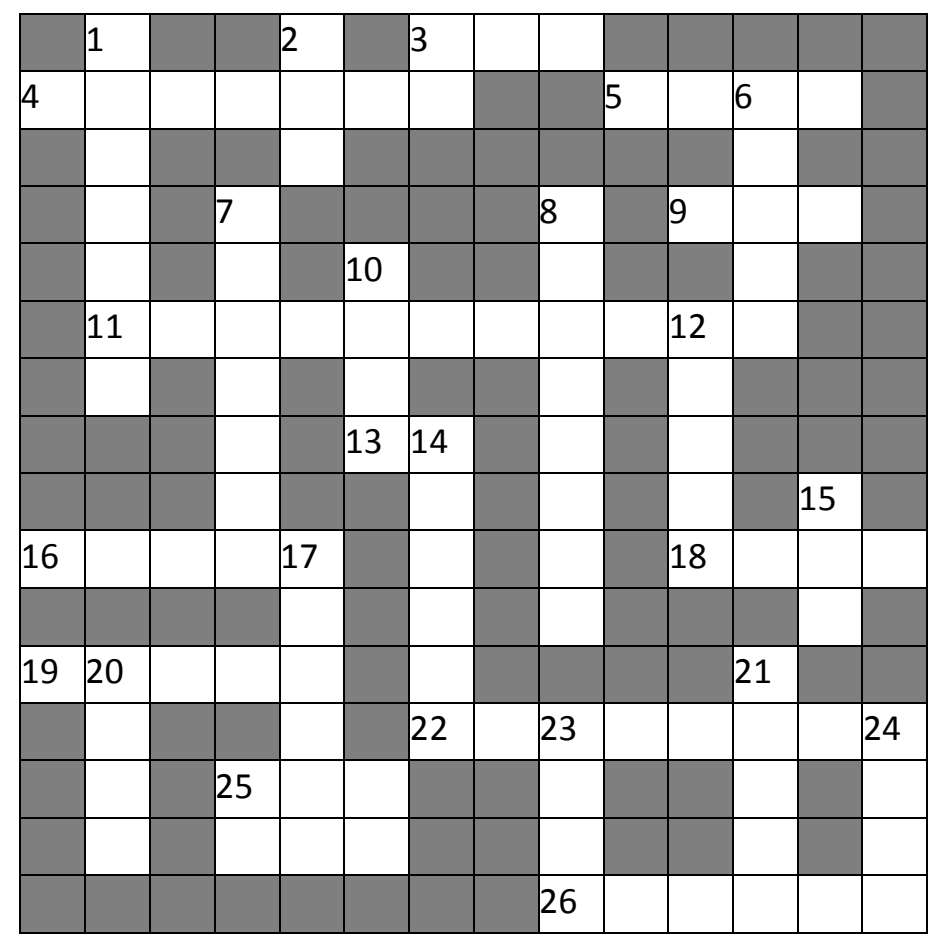

\section{ACROSS}

3. Screening for Thalassemia carriers (abbrv)

4. Former term for faecal incontinence

5. Psychological benefit of breast milk

9. Autism and Asperger's are both classified as

$$
\text { ..s (abbrv) }
$$

11. Cause of Rickets that is most prevalent worldwide

13. A neurodevelopmental disorder that is associated with a high prevalence of ASD (abbrv)

16. Gender affected by Rett syndrome

18. A supraglottic airway that is increasingly used

19. EEG waveform that is excessive in Autism

22. Tuberculosis of spine affects

$$
\text { vertebrae }
$$

25 . Facies of a syndrome characterised by developmental delay but good speech and social skills

26. Denotes suppression of emotions in adults but suppression of activity in neonates

\section{DOWN}

1. Site of lesion in midbrain that causes hyperpyrexia

2. Sexually transmitted infection resulting in increased number of orphans

3. Severe asthma is treated with (abbrv)

6. Junctional rhythm

7. Vitamin A intake affects serum level

8. Used to reduce agitation in children following general anaesthesia

10. A much spoken of approach for managing Autism

12. An EEG waveform that is prominent when awake

14. A traditional Indian system of medicine

15. Prevents rabies (abbrv)

17. Hands and feet in Prader Willi Syndrome

20. The first known oncogenic virus (abbrv)

21. Syndrome that mimics Autistic Spectrum Disorder

23. The most common chronic rheumatologic condition in children

24. Used in developmental assessment of children 